Relations industrielles

Industrial Relations

\title{
IV - Les modalités des réformes de structure Les solutions proposées
}

\section{Marcel Clément}

Volume 3, numéro 8, avril 1948

URI : https://id.erudit.org/iderudit/1023612ar

DOI : https://doi.org/10.7202/1023612ar

Aller au sommaire du numéro

Éditeur(s)

Département des relations industrielles de l’Université Laval

ISSN

0034-379X (imprimé)

1703-8138 (numérique)

Découvrir la revue

Citer cet article

Clément, M. (1948). IV - Les modalités des réformes de structure : les solutions proposées. Relations industrielles / Industrial Relations, 3(8), 114-116.

https://doi.org/10.7202/1023612ar

Tous droits réservés @ C Département des relations industrielles de l’Université Laval, 1948
Ce document est protégé par la loi sur le droit d'auteur. L'utilisation des services d’Érudit (y compris la reproduction) est assujettie à sa politique d'utilisation que vous pouvez consulter en ligne.

https://apropos.erudit.org/fr/usagers/politique-dutilisation/ 


\section{Bulletin des relations industrielles}

Volume 3, numéro 8

Avril 1948

publié par
le Département des relations industrielles de Laval.

T.R.P. Georges-H. LEvesque, o.p., doyen.

Gérard Tremblay, directeur.

Abbé Gérard Dion, secrétaire.

Charles BELANGer et Jean GaGne, assistantssecrétaires.

\section{ABONNEMENT:}

Etranger $\$ 2.00$

Autorisé comme envoi postal de seconde classe, Ministère des Postes, Ottawa.

Adressez toute correspondance au Secrétaire

Département des relations industrielles, Faculté des sciences sociales,

Université Laval,

Québec, P.Q.

\section{SOMMAIRE}

page

Réflexions sur le troisième congrès La Direction..

Les solutions proposées Marcel ClEMENT

La formation professionnelle Gabriel Rousseau.. ..

Les Comités d'entreprises Raymond GÉrin

Clause de renouvellement dans les conventions collectives

G.-M. Groux.

Proposed Solutions

Marcel ClÉMENT.

The renewal clause of collective labour agreements

G.-M. Grroux

Nos collaborateurs

Some reflexions on the third convention

THE EDITORs

\section{LES SOLUTIONS PROPOSÉES}

\section{Marcel CLÉMENT}

Pour faire l'étude, à grands traits, des formes nouvelles de structure de l'entreprise, nous partirons d'une observation particulièrement concrète. Il arrive souvent, lorsque l'or évoque l'instauration d'une coinmunauté du profit dans l'entreprise, que certains se recusent, en alléguant les dangers du paternalisme. On a même vu telle ou telle forme de participation suspectée par des esprits cependant avertis, simplemnet parce qu'ils voient dans cette participation un «cadeau ». (M. l'abbé Dion traitera par ailleurs de l'aspect moral de ce problème). Pour nous, cela nous fait penser à l'attitude d'un homme qui se refuse à marcher de peur de faire un faux pas.

Disons tout net que ce genre de timidité présente des dangers réels. Dans l'intérêt même de la paix sociale, il est nécessaire que le passage d'une structure libérale à une structure communautaire dépende de l'initiative patronale. Un contrat de participation établissant les conditions de celle-ci pour éliminer l'arbitraire de la gratification, confère après sa signature, des droits nouveaux aux salariés. Mais jusqu'à ce moment, ce contrat dé- pend lui-même du bon gré de l'entrepreneur. Dire cela, répéter cela, ce n'est nullement inciter au paternalisme. C'est au contraire inviter les chefs d'entreprise à prendre, en conscience, la mesure de leur responsabilité, au moment où l'initiative dépend d'eux. Il n'est pas assuré qu'il en sera toujours ainsi. Il arrive que la paix sociale dépende de la raison et du bon sens des salariés. Il arrive aussi qu'elle dépende de la loyauté et de la lucidité des patrons. Nommer paternalisme cette loyauté et cette lucidité, c'est jeter une confusion grave dans les esprits, et par conséquent désorienter les meilleures volontés. C'est pourquoi nous avons à plusieurs reprises insisté sur cette conversion intérieure «par laquelle le patron reconnaît dans ses ouvriers des frères dont il est l'aîné responsable ». Une telle droiture d'intention ne posera jamais un acte paternaliste, ce trafic de la confiance ouvrière.

Moyennant quoi, trois règles doivent gouverner toute réforme de structure :

1-En aucun cas, l'établissement d'une modalité de participation ne peut avoir pour résultat 
de mettre en discussion le salaire garanti qu'il soit égal ou supérieur au mininum vital.

2-La préférençe accordée à telle ou telle modalité de participation doit principalement dépendre des dimensions et des particularités de chaque entreprise, même s'il apparaît qu'en général, tel ou tel système est préférable.

3-Il faut aller plus loin encore dans la liberté. La communauté de profit ne doit pas être un * vêtement de confection » mais un \&vêtement fait sur mesure». Ainsi, des modifications, des additions pourront être apportées à chaque formulc pour tenir compte d'une psychologie professionnelle particulière, d'une technique de fabrication nouvelle etc. On a vu aussi l'exemple de deux modalités être combinées entre elles. Ce qui importe, c'est l'intention d'équité et le réalisme de son extécution, - non le formalisme de la « recette» utilisée.

Ces points retenus, nous vous proposons de faire une revue, à la fois brève et claire, des diverses modalités qui ont été jusqu'ici tentées avec succès.

\section{1-La participation aux bénéfices}

\section{(Système Romanet)}

La participation aux bénéfices est une vieille chose dont les inconvénients ont été souvent énumérés, et dont on sait maintenant que ces inconvénients n'était pas ceux du système lui-mème, mais de l'esprit - dans lequel il tait traditionnellement appliqué. C'est donc, non des contrefaçons légitimement criticables dans leur principe même, mais de la véritable participation que nous entendons parler; de celle, par exemple, dont M. Romanet, et derrière lui, de nombreux industriels d'Europe, ont montré qu'elle était possible et qu'elle était féconde.

M. Romanet est un homme qui inspire confiance. C'est à lui que l'on doit la trouvaille de la Caisse de compensation qui a permis l'instauration en France, des allocations familiales, Pour obtenir la généralisation de celles-ci dans toutes les entreprises, M. Romanet a prouvé le mouvement en marchant: il a personnellement donné l'exemple en fondant la première caisse de compensation avec certains de ses amis. Et le résultat a dépassé les espérances. Le renouveau familial en France a été facilité par cette mesure, devenue obligatoire, et dont la portée morale est ainsi incalculable.

M. Romanet connaîtra-t-il, à longue échéance, un succès aussi exceptionnel avec l'autre grande initiative de sa vie de patron: la participation des salariés aux résultats obtenus dans l'entreprise ? Il n'est pas interdit de le penser, si l'on songe qu'il applique dans son entreprise, et avec un bonheur constant, la même formule depuis 1927, c'est-à-dire depuis vingt et un ans: l'âge de la majorité!

M. Romanet part de l'observation des faits. Il considére que les facteurs de l'entreprise sont:

-le capital, nécessaire pour permettre l'acquisition des immeubles: terrains, bâtiments et des meubles: outillages, matière première, et assurer le fond de roulement de l'entreprise.

-le travail, comprenant:

a-le personnel de direction, qui assure le gouvernement de l'entreprise par le choix et la mise en oeuvre des moyens propres à réaliser la fin désirée;

b-le personnel d'exécution, qui, unissant l'intérêt de l'esprit et l'effort du corps, assure l'adaptation de la matière à la satisfaction des besoins humains.

A chacun de ces facteurs reviennent trois sortes de rémunération:

-un salaire normal, ou salaire de base;

-un salaire ajusté, ou salaire de prévoyance;

-un boni variable, ou participation au bénéfice.

Après inventaire de clôture, le profit est distribué de la manière suivante: 5 à $10 \%$ prélevés pour la fraction communautaire de solidarité - le reste réparti entre le capital et le travail.

Ainsi, le capital perçoit:

1-un intérêt normal, prenant pour base, par exemple, le taux courant;

2-une réserve annuelle, qui va à un fonds de prévoyance, en vue de pourvoir aux déficits et aux risques éventuels;

3-un boni, c'est-à-dire une part du bénéfice net tel qu'il ressort du bilan.

De son côté le travail - personnel de direction, personnel d'exécution - perçoit:

1-un salaire normal, rétribution de base tenant compte de la qualification professionnelle et des aptitudes particulières;

2-un salaire ajusté, prévu ou non par les dispositions de la loi: allocations familiales, congés payés, assurances contre les risques de l'existence;

3-un boni, c'est-à-dire une part du bénéfice net tel qu'il ressort du bilan. 
La répartition du boni est nécessairement la partie la plus délicate de l'opération. Nous empruntons à $\mathrm{M}$. Paul Chas ${ }^{1}$ l'analyse suivante, remarquable de clarté, selon les trois hypothèses qu'il envisage:

1-Si la totalité du bénéfice net est disponible, il est réparti entre les facteurs de la production au prorata des sommes qu'ils ont touchées pendant l'année au titre salaire. Toutefois, pour récompenser le rendement, la valeur, les responsabilités encourues, les sommes attribuées au personnel peuvent être affectés d'un coefficient de majoration, un coefficient spécial permettant de tenir compte de lancienneté et des charges de famille. De même, l'intérèt du capital peut-être majoré si le risque de l'affaire est important et si les possesseurs du capital sont en même temps les animateurs de l'entreprise.

2-Si les bénéfices se traduisent partie en espèces disponibles, partie en achat de terrains, bâtiments, machines, matières en magasin, fonds de roulement complèmentaire, la partie disponible est distribuée comme il est dit ci-dessus. Quant à l'autre partie elle fait l'objet d'une inscription sur des «carnets de boni » individuels. La part de chaque bénéficiaire-actionnaire ou membre du personnel participant ${ }^{2}$ est calculée avec les mêmes coefficients que pour les attributions en espèces. Elle devient une part de capital portant intérêt, et ne pouvant être versée que si les possibilités de l'entreprise le permettent, ou dans certains cas de force majeure.

(1) P. Chas: La rémunération du travail, dans "Chronique sociale de France" de janvier 1946.

(2) Les membres du personnel ayant moins de deux ans d'ancienneté n'ont pas droit à cette rémunération à moins que leur valeur professionnelle les classe, dès leur arrivée, comme générateur de bénéfices.
3-Enfin, s'il y a perte sur un exercice, elle est comblée par un prélèvement effectué d'abord sur la réserve constituée à cet effet, puis sur le boni inscrit au carnet de chacun des participants ${ }^{3}$ et enfin, sur le capital lui-mème, s'il y a lieu. Par la suite, à la fin du premier exercice comportant des bénéfices, on redressera la situation du capital pour la ramener à son chiffre primitif. Le redressement effectué, les trois facteurs: capital, direction, travail, se partageront à nouveau les bénéfices, soit sous forme de distribution en espèce, soit sous forme d'inscription au carnet de boni.

Telles sont les lignes générales de la formule. Il va de soi qu'elle suppose que l'équité et la prudence inspirent les déterminations des divers pourcentages, et que la parfaite sincérité des comptes soit réalisée et évidente aux yeux de tous. Dans ce dessein, M. Romanet en a remis le contrôle aux mains de commissaires assermentés. La confiance a répondu à son attente puisque l'on a vu des travailleurs demander spontanément à investir dans l'entreprise sous forme d'actions ou de dépôts inscrits au carnet de boni, les bénéfices qui leur sont dus. ${ }^{4}$

Pour terminer, signalons qu'à l'heure présente, pour l'ensemble du territoire français, plus de quatre-vingt-dix firmes, de caractères très différents et d'importance très inégale, ont adopté ce régime et s'en déclarent pleinement satisfaits. C'est vraiment là «tempérer le contrat de travail par des éléments empruntés au contrat de société».

(3) Au lieu de faire retrait du carnet de boni, on peut ouvrir un compte "pertes à amortir" que les bénéfices futurs éteindront par priorité.

(4) Pour plus de détail, lire E. Romanet: "Participation des salariés aux résultats obtenus dans les entreprises", chez l'auteur, 17 cours Jean Jeaurès, Grenoble (Isère), France.

\section{LA FORMATION PROFESSIONNELLE}

\section{Gabriel ROUSSEAU}

Les parents sont responsables de l'éducation de leurs enfants. L'autorité religieuse ou civile ne doit intervenir que pour faciliter aux parents l'exercice de ce droit.

La formation professionnelle a pour premier objectif de préparer un nombre suffisant mais non excessif de travailleurs de chaque catégorie et de chaque type, nécessaires à l'économie nationale et capables d'accomplir collectivement, de la manière la plus efficace, les tâches qui leur seront dévolues dans l'entreprise.

Le rôle des parents dans la formation professionnelle doit être d'orienter et de diriger l'enfant dans le choix d'une carrière, et de confier aux institutions qui ont autorité pour déterminer les conditions d'admission à la profession le soin de

(1) Résumés de quelques conférences prononcées lors du 3ième Congrès des relations industrielles de Laval. 